\title{
OpenAttack: An Open-source Textual Adversarial Attack Toolkit
}

\author{
Guoyang Zeng ${ }^{1,2 *}$, Fanchao Qi ${ }^{1,2 *}$, Qianrui Zhou ${ }^{1,2}$, Tingji Zhang ${ }^{1,2}$, Zixian $\mathbf{M a}^{4 \dagger}$, \\ Bairu Hou $^{2,5}$, Yuan Zang ${ }^{1,2}$, Zhiyuan Liu ${ }^{1,2,3 \ddagger}$, Maosong Sun ${ }^{1,2,3}$ \\ ${ }^{1}$ Department of Computer Science and Technology, Tsinghua University, Beijing, China \\ ${ }^{2}$ Beijing National Research Center for Information Science and Technology \\ ${ }^{3}$ Institute for Artificial Intelligence, Tsinghua University, Beijing, China \\ ${ }^{4}$ Stanford University ${ }^{5}$ School of Economics and Management, Tsinghua University \\ zenggy@mail.tsinghua.edu.cn, qfol7@mails.tsinghua.edu.cn
}

\begin{abstract}
Textual adversarial attacking has received wide and increasing attention in recent years. Various attack models have been proposed, which are enormously distinct and implemented with different programming frameworks and settings. These facts hinder quick utilization and fair comparison of attack models. In this paper, we present an opensource textual adversarial attack toolkit named OpenAttack to solve these issues. Compared with existing other textual adversarial attack toolkits, OpenAttack has its unique strengths in support for all attack types, multilinguality, and parallel processing. Currently, OpenAttack includes 15 typical attack models that cover all attack types. Its highly inclusive modular design not only supports quick utilization of existing attack models, but also enables great flexibility and extensibility. OpenAttack has broad uses including comparing and evaluating attack models, measuring robustness of a model, assisting in developing new attack models, and adversarial training. Source code and documentation can be obtained at https://github.com/thunlp/ openAttack.
\end{abstract}

\section{Introduction}

Deep neural networks (DNNs) have been found to be susceptible to adversarial attacks (Szegedy et al., 2014; Goodfellow et al., 2015). The attacker uses adversarial examples, which are maliciously crafted by imposing small perturbations on original input, to fool the victim model. With the wide application of DNNs to practical systems accompanied by growing concern about their security, research on adversarial attacking has become increasingly important. Moreover, adversarial attacks are also

\footnotetext{
${ }^{*}$ Indicates equal contribution

${ }^{\dagger}$ Work done during internship at Tsinghua University

${ }^{\ddagger}$ Corresponding author. Email: liuzy@tsinghua.edu.cn
}

helpful to improve robustness and interpretability of DNNs (Wallace et al., 2019a).

In the field of natural language processing (NLP), diverse adversarial attack models have been proposed (Zhang et al., 2020). These models vary in accessibility to the victim model (ranging from having full knowledge to total ignorance) and perturbation level (character-, word- or sentence-level). In addition, they are originally proposed to attack different victim models on different NLP tasks under different evaluation protocols.

This immense diversity causes serious difficulty for fair and apt comparison between different attack models, which is unfavourable to the development of textual adversarial attacking. Further, although most attack models are open-source, they use different programming frameworks and settings, which lead to unnecessary time and effort when implementing them.

To tackle these challenges, a textual adversarial attacking toolkit named TextAttack (Morris et al., 2020) has been developed. It implements several textual adversarial attack models under a unified framework and provides interfaces for utilizing existing attack models or designing new attack models. So far, TextAttack has attracted considerable attention and facilitated the birth of new attack models such as BAE (Garg and Ramakrishnan, 2020).

In this paper, we present OpenAttack, which is also an open-source toolkit for textual adversarial attacking. Similar to TextAttack, OpenAttack adopts modular design to assemble various attack models, in order to enable quick implementation of existing or new attack models. But OpenAttack is different from and complementary to TextAttack mainly in the following three aspects:

(1) Support for all attacks. TextAttack utilizes a relatively rigorous framework to unify different attack models. However, this framework is naturally not suitable for sentence-level adversarial attacks, 


\begin{tabular}{l|cc|l}
\hline \multicolumn{1}{c|}{ Model } & Accessibility & Perturbation & \multicolumn{1}{c}{ Main Idea } \\
\hline SEA (Ribeiro et al., 2018) & Decision & Sentence & Rule-based paraphrasing \\
SCPN (Iyyer et al., 2018) & Blind & Sentence & Paraphrasing \\
GAN (Zhao et al., 2018) & Decision & Sentence & Text generation by encoder-decoder \\
TextFooler (Jin et al., 2020) & Score & Word & Greedy word substitution \\
PWWS (Ren et al., 2019) & Score & Word & Greedy word substitution \\
Genetic (Alzantot et al., 2018) & Score & Word & Genetic algorithm-based word substitution \\
SememePSO (Zang et al., 2020) & Score & Word & Particle swarm optimization-based word substitution \\
BERT-ATTACK (Li et al., 2020) & Score & Word & Greedy contextualized word substitution \\
BAE (Garg and Ramakrishnan, 2020) & Score & Word & Greedy contextualized word substitution and insertion \\
FD (Papernot et al., 2016b) & Gradient & Word & Gradient-based word substitution \\
TextBugger (Li et al., 2019) & Gradient, Score & Word+Char & Greedy word substitution and character manipulation \\
UAT (Wallace et al., 2019a) & Gradient & Word, Char & Gradient-based word or character manipulation \\
HotFlip (Ebrahimi et al., 2018) & Gradient & Word, Char & Gradient-based word or character substitution \\
VIPER (Eger et al., 2019) & Blind & Char & Visually similar character substitution \\
DeepWordBug (Gao et al., 2018) & Score & Char & Greedy character manipulation \\
\hline
\end{tabular}

Table 1: Textual adversarial attack models involved in OpenAttack, among which the three sentence-level models SEA, SCPN and GAN together with FD, UAT and VIPER are not included in TextAttack for now. "Accessibility" is the accessibility to the victim model, and "Perturbation" refers to perturbation level. "Sentence", "Word" and "Char" denote sentence-, word- and character-level perturbations. In the columns of Accessibility and Perturbation, "A, B" means that the attack model supports both A and B, while "A+B" means that the attack model conducts A and B simultaneously.

an important and typical kind of textual adversarial attacks. Thus, no sentence-level attack models are included in TextAttack. In contrast, OpenAttack adopts a more flexible framework that supports all types of attacks including sentence-level attacks.

(2) Multilinguality. TextAttack only covers English textual attacks while OpenAttack supports English and Chinese now. And its extensible design enables quick support for more languages.

(3) Parallel processing. Running some attack models maybe very time-consuming, e.g., it takes over 100 seconds to attack an instance with the SememePSO attack model (Zang et al., 2020). To address this issue, OpenAttack additionally provides support for multi-process running of attack models to improve attack efficiency.

Moreover, OpenAttack is fully integrated with HuggingFace's transformers ${ }^{1}$ and datasets ${ }^{2}$ libraries, which allows convenient adversarial attacks against thousands of NLP models (especially pre-trained models) on diverse datasets. OpenAttack also has great extensibility. It can be easily used to attack any customized victim model, regardless of the used programming framework (PyTorch, TensorFlow, Keras, etc.), on any customized dataset.

OpenAttack can be used to (1) provide vari-

\footnotetext{
${ }^{1}$ https://github.com/huggingface/ transformers

${ }^{2}$ https://github.com/huggingface/ datasets
}

ous handy baselines for attack models; (2) comprehensively evaluate attack models using its thorough evaluation metrics; (3) assist in quick development of new attack models; (4) evaluate the robustness of an NLP model against various adversarial attacks; and (5) conduct adversarial training (Goodfellow et al., 2015) to improve model robustness by enriching the training data with generated adversarial examples.

Recent years have witnesses the rapid development of adversarial attacks in computer vision (Akhtar and Mian, 2018), which is promoted by many visual attack toolkits such as CleverHans ( $\mathrm{Pa}-$ pernot et al., 2018), Foolbox (Rauber et al., 2017), AdvBox (Goodman et al., 2020), etc. We hope OpenAttack, together with TextAttack and other similar toolkits, can play a constructive role in the development of textual adversarial attacks.

\section{Formalization and Categorization of Textual Adversarial Attacking}

We first formalize the task of textual adversarial attacking for text classification, and the following formalization can be trivially adapted to other NLP tasks. For a given text sequence $x$ that is correctly classified as its ground-truth label $y$ by the victim model $F$, the attack model $A$ is supposed to transform $x$ into $\hat{x}$ by small perturbations, whose ground-truth label is still $y$ but classification result given by $F$ is $\hat{y} \neq y$. Next, we introduce the catego- 
rization of textual adversarial attack models from three perspectives.

According to the attack model's accessibility to the victim model, existing attack models can be categorized into four classes, namely gradientbased, score-based, decision-based and blind models. First, gradient-based attack models are also called white-box attack models, which require full knowledge of the victim model to conduct gradient update. Most of them are inspired by the fast gradient sign method (Goodfellow et al., 2015) and forward derivative method (Papernot et al., 2016a) in visual adversarial attacking.

In contrast to white-box attack models, blackbox models do not need to have complete information on the victim model, and can be subcategorized into score-based, decision-based and blind models. Blind models are ignorant of the victim model at all. Score-based models require the prediction scores (e.g., classification probabilities) of the victim model, while decision-based models only need the final decision (e.g., predicted class).

According to the level of perturbations imposed on original input, textual adversarial attack models can be classified into sentence-level, wordlevel and character-level models. Sentence-level attack models craft adversarial examples mainly by adding distracting sentences (Jia and Liang, 2017), paraphrasing (Iyyer et al., 2018; Ribeiro et al., 2018) or text generation by encoder-decoder (Zhao et al., 2018). Word-level attack models mostly conduct word substitution, namely substituting some words in the original input with semantically identical or similar words such as synonyms (Jin et al., 2020; Ren et al., 2019; Alzantot et al., 2018). Some word-level attack models also use operations including deleting and adding words (Zhang et al., 2019; Garg and Ramakrishnan, 2020). Characterlevel attack models usually carry out character manipulations including swap, substitution, deletion, insertion and repeating (Eger et al., 2019; Ebrahimi et al., 2018; Belinkov and Bisk, 2018).

Finally, adversarial attack models can also be categorized into targeted and untargeted models based on whether the wrong classification result given by the victim model $(\hat{y})$ is pre-specified (mainly for the multi-class classification models). Most existing attack models support (by minor adjustment) both targeted and untargeted attacks, and we give no particular attention to this attribute of attack models in this paper.

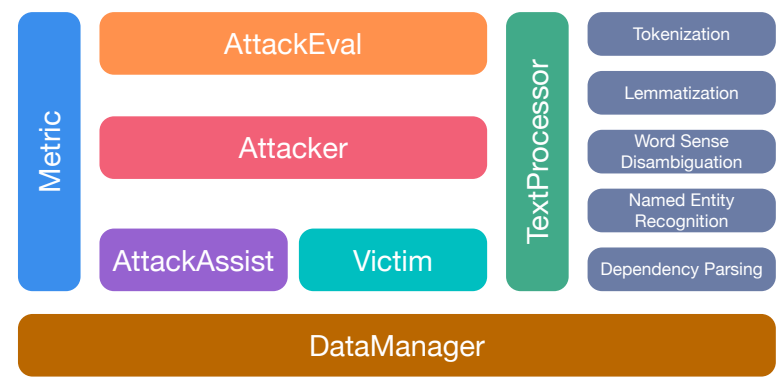

Figure 1: Overall architecture of OpenAttack.

Currently OpenAttack includes 15 different attack models, which cover all the victim model accessibility and perturbation level types. Table 1 lists the attack models involved in OpenAttack.

\section{Toolkit Design and Architecture}

In this section, we describe the design philosophy and modular architecture of OpenAttack.

We extract and properly reorganize the commonly used components from different attack models, so that any attack model can be assembled by them. Considering the significant distinctions among different attack models, especially those between the sentence-level and word/char-level attack models, it is hard to embrace all attack models within a unified framework like TextAttack. Therefore, we leave considerable freedom for the skeleton design of attack models, and focus more on streamlining the general processing of adversarial attacking and providing common components used in attack models. Next we introduce the modules of OpenAttack one by one, and Figure 1 illustrates an overview of all the modules.

- TextProcessor. This module is aimed at processing the original input so as to assist attack models in generating adversarial examples. It consists of several functions used for tokenization, lemmatization, delemmatization, word sense disambiguation (WSD), named entity recognition (NER) and dependency parsing. Currently it supports English and Chinese, and support for other languages can be realized simply by rewriting the TextProcessor base class.

- Victim. This module wraps the victim model. It supports both neural network-based model implemented by any programming framework (especially the HuggingFace's transformers) and traditional machine learning model. It is mainly composed of three functions that are used to 
obtain the gradient with respect to the input, prediction scores and predicted class of a victim model.

- Attacker. This is the core module of OpenAttack. It comprises various attack models and can generate adversarial examples for given input against a victim model.

- AttackAssist. This is an assistant module of Attacker. It mainly packs different word and character substitution methods that are widely used in word- and character-level attack models. Attacker queries this module to get substitutions for a word or character. Now it includes word embedding-based (Alzantot et al., 2018; Jin et al., 2020), synonym-based (Ren et al., 2019) and sememe-based (Zang et al., 2020) word substitution methods, and visual character substitution method (Eger et al., 2019). In addition, some useful components used in sentencelevel attack models are also included, such as paraphrasing based on back-translation.

- Metric. This module provides several adversarial example quality metrics which can serve as either the constraints on the adversarial examples during attacking or evaluation metrics for evaluating adversarial attacks. It currently includes following metrics: (1) language model prediction score for a given word in a context given by Google one-billion words language model (Jozefowicz et al., 2016) (this metric can be used as the constraint on adversarial examples only); (2) word modification rate, the percentage of modified words of an adversarial example compared with the original example; (3) formal similarity between the adversarial example and original example, which is measured by Levenshtein edit distance (Levenshtein, 1966), character- and word-level Jaccard similarity (Jaccard, 1912) and BLEU score (Papineni et al., 2002); (4) semantic similarity between the adversarial example and original example measured by Universal Sentence Encoder (Cer et al., 2018) and Sentence Transformers (Reimers and Gurevych, 2019); (5) adversarial example fluency measured with perplexity computed by GPT-2 (Radford et al., 2019); and (6) grammaticality measure by the grammatical errors given by LanguageTool. ${ }^{3}$

\footnotetext{
${ }^{3}$ https: / www. languagetool.org
}

\begin{tabular}{c|c|l}
\hline Perspective & Metric & Better? \\
\hline Attack & Attack Success Rate & Higher \\
Effectiveness & Word Modification Rate & Lower \\
\hline & Formal Similarity & Higher \\
Adversarial & Semantic Similarity & Higher \\
Example & Fluency (GPT-2 perplexity) & Lower \\
Quality & Grammaticality (Grammatical Errors) & Lower \\
& Average Victim Model Query Times & Lower \\
Attack & Average Running Time & Lower \\
Efficiency & &
\end{tabular}

Table 2: Evaluation metrics in OpenAttack. "Higher" and "Lower" mean the higher/lower the metric is, the better an attack model performs.

- AttackEval. This module is used to evaluate textual adversarial attacks from different perspectives including attack effectiveness, adversarial example quality and attack efficiency: (1) the attack effectiveness metric is attack success rate, the percentage of the attacks that successfully fool the victim model; (2) adversarial example quality is measured by the last five metrics in the Metric module; and (3) attack efficiency has two metrics including average victim model query times and average running time of attacking one instance. Table 2 lists all the evaluation metrics in OpenAttack.

The realization of multi-processing is incorporated in this module, with the help of Python multiprocessing library. In addition, this module can also visualize and save attack results, e.g., display original input and adversarial examples and emphasize their differences.

- DataManager. This module manages all the data as well as saved models that are used in other modules. It supports accessing and downloading data/models. Specifically, it deals with the data used in the AttackAssist module such as character embeddings, word embeddings and WordNet synonyms, the models used in the TextProcessor module such as NER model and dependency parser, the built-in trained victim models, and auxiliary models used in Attacker such as the paraphrasing model for the paraphrasing-based attack models. This module helps efficiently and handily utilize data.

\section{Toolkit Usage}

OpenAttack provides a set of easy-to-use interfaces that can meet almost all the needs in textual adversarial attacking, such as preprocessing text, generating adversarial examples to attack a victim 


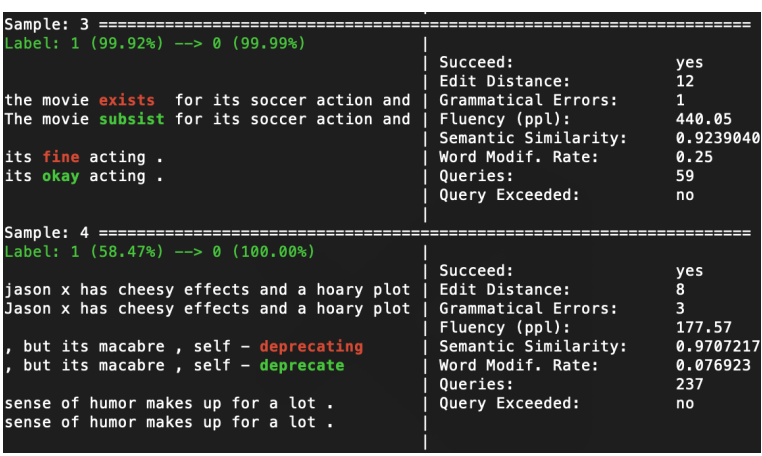

Figure 2: Part of attack results for individual instances.

model and evaluating attack models. Moreover, OpenAttack has great flexibility and extensibility and supports easy customization of victim models and attack models. Next, we showcase some basic usages of OpenAttack.

\subsection{Built-in Attack and Evaluation}

OpenAttack builds in some commonly used NLP models such as LSTM (Hochreiter and Schmidhuber, 1997) and BERT (Devlin et al., 2019) that have been trained on commonly used NLP datasets. Users can use the built-in victim models to quickly conduct adversarial attacks. The following code snippet shows how to use Genetic (Alzantot et al., 2018) to attack BERT on the test set of SST-2 (Socher et al., 2013) with 4-process parallel running:

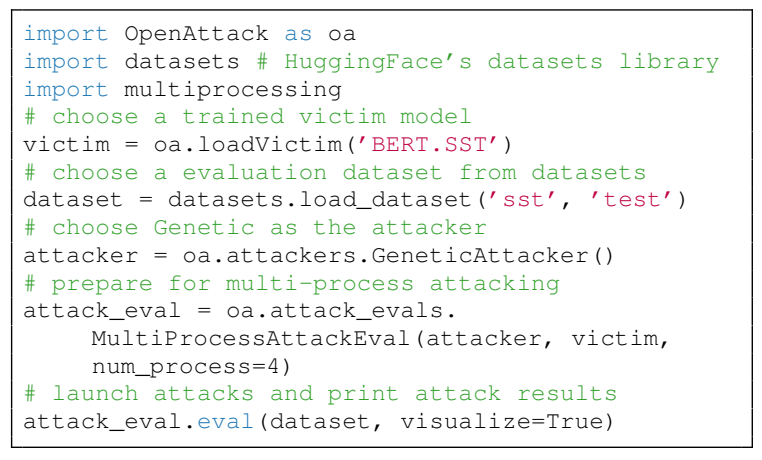

Figure 2 displays the printed attack results for individual instances from above code. We can see OpenAttack prints the original input as well as the word-aligned adversarial example, where the perturbed words are colored. In addition, a series of attack evaluation results are listed. At the end of individual attack results, OpenAttack provides an attack summary composed of average evaluation results of specified metrics among all instances, as shown in Figure 3.

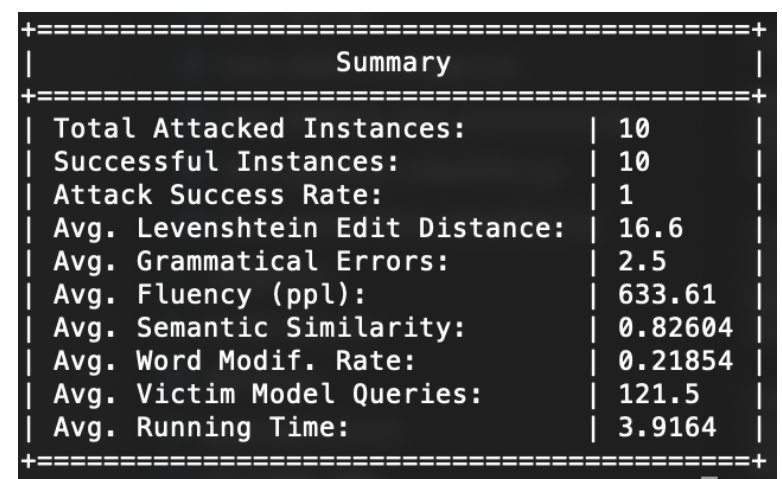

Figure 3: Summary of attack results.

\subsection{Customized Victim Models}

It is very common for users to launch attacks against their own models that have been trained on specific datasets, particularly when evaluating the robustness of a victim model. It is impossible to exhaustively build in all victim models. Thus, easy customization for victim models is very important.

OpenAttack provides simple and convenient interfaces for victim model customization. For a trained model implemented with whichever programming framework, users just need to configure some model access interfaces that provide accessibility required for the attack model under the Victim class. The following code snippet shows how to use Genetic to attack a customized sentiment analysis model, a statistical model in NLTK (Bird et al., 2009), on the test set of SST.

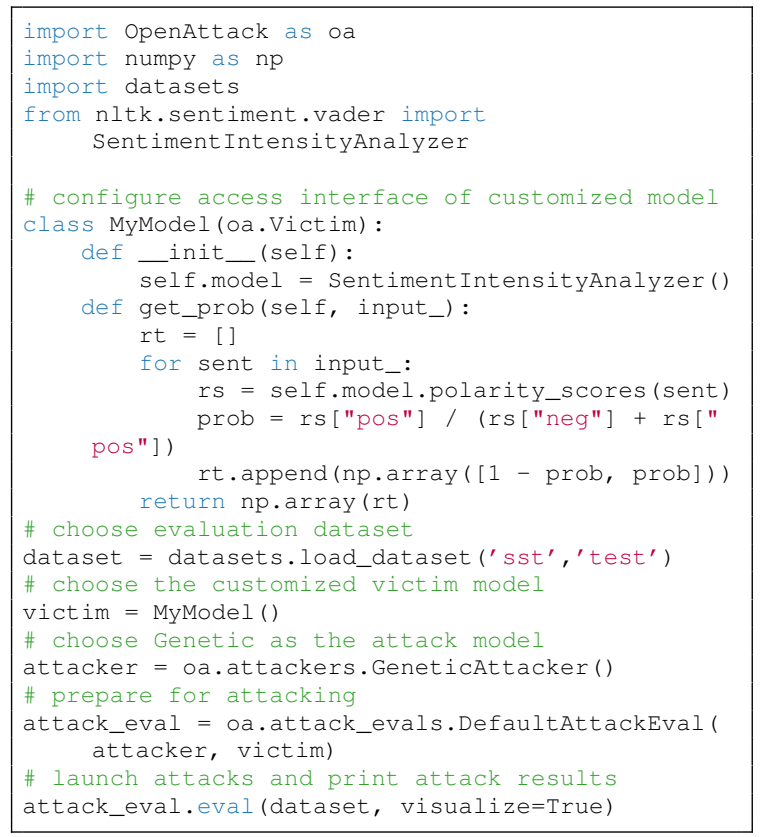

In addition, OpenAttack supports easy customization of attack models thanks to its inclusive 


\begin{tabular}{|c|c|c|c|c|c|c|c|c|c|c|c|c|}
\hline \multirow{2}{*}{ Model } & \multicolumn{2}{|c|}{ Type } & \multirow{2}{*}{$\frac{\text { Effectiveness }}{\text { ASR }}$} & \multicolumn{5}{|c|}{ Adversarial Example Quality } & \multicolumn{4}{|c|}{ Attack Efficiency } \\
\hline & Accessibility & Perturbation & & WMR & LES & SemSim & Fluency & Grm & \#Query & $\mathrm{T}_{1}$ & $\mathrm{~T}_{2}$ & $\mathrm{~S}$ \\
\hline SEA & Decision & Sentence & 0.12 & - & 14.7 & 0.90 & 398 & 2.2 & 2.0 & 37.1 & - & - \\
\hline SCPN & Blind & Sentence & 0.68 & - & 55.6 & 0.56 & 432 & 2.7 & 11.0 & 3.58 & 2.30 & 1.56 \\
\hline GAN & Decision & Sentence & 0.41 & - & 68.8 & 0.26 & 512 & 4.2 & 2.0 & 0.60 & 2.00 & 0.30 \\
\hline TextFooler & Score & Word & 0.90 & 0.11 & 14.1 & 0.87 & 621 & 4.6 & 130.5 & 5.75 & 3.25 & 1.77 \\
\hline PWWS & Score & Word & 0.78 & 0.20 & 17.9 & 0.84 & 613 & 2.9 & 124.8 & 5.26 & 2.88 & 1.83 \\
\hline Genetic & Score & Word & 0.36 & 0.11 & 13.4 & 0.88 & 689 & 4.7 & 242.1 & 54.11 & 27.56 & 1.96 \\
\hline SememePSO & Score & Word & 0.82 & 0.14 & 2.9 & 0.89 & 711 & 2.9 & 177.9 & 102.44 & 52.41 & 1.95 \\
\hline BERT-ATTACK & Score & Word & 0.87 & 0.31 & 4.2 & 0.86 & 796 & 4.4 & 51.9 & 2.38 & 1.57 & 1.51 \\
\hline BAE & Score & Word & 0.77 & 0.68 & 5.4 & 0.82 & 1147 & 4.3 & 103.0 & 2.97 & 1.79 & 1.66 \\
\hline FD & Gradient & Word & 0.16 & 0.24 & 17.9 & 0.85 & 908 & 3.1 & 10.9 & 34.57 & 28.36 & 1.22 \\
\hline TextBugger & Gradient & Word+Char & 0.25 & 0.15 & 10.6 & 0.61 & 512 & 7.1 & 150.0 & 8.49 & 4.37 & 1.94 \\
\hline UAT & Gradient & Word & 0.43 & 0.15 & 24.0 & 0.85 & 620 & 2.8 & 2.0 & 0.08 & - & - \\
\hline HotFlip & Gradient & Word & 0.47 & 0.08 & 8.9 & 0.93 & 333 & 2.7 & 105.4 & 2.77 & 1.82 & 1.52 \\
\hline VIPER & Blind & Char & 0.27 & - & 24.2 & 0.22 & 347 & 15.8 & 3.0 & 4.01 & 2.04 & 1.97 \\
\hline DeepWordBug & Score & Char & 0.46 & - & 7.9 & 0.73 & 731 & 6.1 & 22.0 & 0.97 & 0.62 & 1.56 \\
\hline
\end{tabular}

Table 3: Evaluation results of different attack models when attacking BERT on SST-2. ASR = Attack Success Rate, WMR = Word Modification Rate that is only applicable to word-level attacks, LES $=$ Levenshterin Edit Distance, SemSim = Semantic Similarity measured by Universal Sentence Encoder, Fluency = GPT-2 perplexity, $\mathrm{Grm}=$ number of grammatical errors, \#Query = average victim model query times, $\mathrm{T}_{1}$ and $\mathrm{T}_{2}$ represent average running time of attacking one instance (seconds) with single and dual process, $\mathrm{S}=\mathrm{T}_{1} / \mathrm{T}_{2}$ is speedup. Notice that it is meaningless to run SEA and UAT with multi-process because they learn and conduct global perturbations.

modular design. Due to limited space, please visit the GitHub project page for more examples including attacking HuggingFace's pre-trained models, using customized evaluation metrics and conducting adversarial training. ${ }^{4}$

\section{Evaluation}

In this section, we conduct evaluations for all the attack models included in OpenAttack.

We use SST-2 as the evaluation dataset and

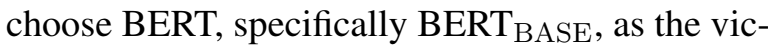
tim model. After fine-tuning on the training set, BERT achieves 90.31 accuracy on the test set. Due to great diversity of attack models, it is hard to impose many constraints on attacks like previous work that focuses on a specific kind of attack. We only restrict the maximum victim model query times to 500. In addition, to improve evaluation efficiency, we randomly sample 1,000 correctly classified instances from the test set as the original input to be perturbed. We use the original default hyper-parameter settings of all attack models.

Table 3 shows the evaluation results. By comparison with originally reported results, we confirm the correctness of our implementation. We also observe that multi-processing can effectively improve attack efficiency of most attack models (the speedup is greater than 1). For some very efficient attack models whose average running time is quite

\footnotetext{
${ }^{4}$ https://github.com/thunlp/OpenAttack/ tree/master/examples
}

short (like GAN), the additional time cost from multi-processing may reduce efficiency instead.

\section{Related Work}

There have been quite a few open-source libraries of generating adversarial examples for continuous data, especially images, such as CleverHans (Papernot et al., 2018), Foolbox (Rauber et al., 2017), Adversarial Robustness Toolbox (ART) (Nicolae et al., 2018) and AdvBox (Goodman et al., 2020). These libraries enable practitioners to easily make adversarial attacks with different methods and have greatly facilitated the development of adversarial attacking for continuous data.

As for discrete data, particularly text, there exist few adversarial attack libraries. As far as we know, TextAttack (Morris et al., 2020) is the only such library. It utilizes a relatively rigorous framework to unify many attack models and provides interfaces for using the existing attack models or designing new attack models. As mentioned in Introduction, our OpenAttack is mainly different from and complementary to TextAttack in allattack-type support, multilinguality and parallel processing.

There are also some other toolkits concerned with textual adversarial attacking. TEAPOT (Michel et al., 2019) is an open-source toolkit to evaluate the effectiveness of textual adversarial examples from the perspective of preservation of meaning. It is mainly designed for the attacks 
against sequence-to-sequence models, but can also be geared towards text classification models. AllenNLP Interpret (Wallace et al., 2019b) is a framework for explaining the predictions of NLP models, where adversarial attacking is one of its interpretation methods. It focuses on interpretability of NLP models and only incorporates two attack models.

\section{Conclusion and Future Work}

In this paper, we present OpenAttack, an opensource textual adversarial attack toolkit that provides a wide range of functions in textual adversarial attacking. It is a great complement to existing counterparts because of its unique strengths in allattack-type support, multilinguality and parallel processing. Moreover, it has great flexibility and extensibility and provides easy customization of victim models and attack models. In the future, we will keep OpenAttack updated to incorporate more up-to-date attack models and support more functions to facilitate the research on textual adversarial attacks.

\section{Acknowledgements}

This work is supported by the National Key Research and Development Program of China (Grant No. 2020AAA0106501 and No. 2020AAA0106502) and Beijing Academy of Artificial Intelligence (BAAI). We also thank all the anonymous reviewers for their valuable comments and suggestions.

\section{Broader Impact Statement}

There is indeed a probability that OpenAttack is misused to maliciously attack some NLP systems. But we believe that we should face up to the potential risks of adversarial attacks rather than pretend not to notice them. As the development of adversarial learning in computer vision, the studies on adversarial attacks actually promote the studies on adversarial defenses and model robustness. We hope more people in the NLP community can realize the robustness issue and OpenAttack can play a constructive role.

\section{References}

Naveed Akhtar and Ajmal Mian. 2018. Threat of adversarial attacks on deep learning in computer vision: A survey. Ieee Access, 6:14410-14430.
Moustafa Alzantot, Yash Sharma, Ahmed Elgohary, Bo-Jhang Ho, Mani Srivastava, and Kai-Wei Chang. 2018. Generating natural language adversarial examples. In Proceedings of the EMNLP.

Yonatan Belinkov and Yonatan Bisk. 2018. Synthetic and natural noise both break neural machine translation. In Proceedings of ICLR.

Steven Bird, Ewan Klein, and Edward Loper. 2009. Natural language processing with Python: analyzing text with the natural language toolkit. O'Reilly Media, Inc.

Daniel Cer, Yinfei Yang, Sheng-yi Kong, Nan Hua, Nicole Limtiaco, Rhomni St John, Noah Constant, Mario Guajardo-Cespedes, Steve Yuan, Chris Tar, et al. 2018. Universal sentence encoder for english. In Proceedings of EMNLP.

Jacob Devlin, Ming-Wei Chang, Kenton Lee, and Kristina Toutanova. 2019. BERT: Pre-training of deep bidirectional Transformers for language understanding. In Proceedings of NAACL-HLT.

Javid Ebrahimi, Anyi Rao, Daniel Lowd, and Dejing Dou. 2018. Hotflip: White-box adversarial examples for text classification. In Proceedings of $A C L$.

Steffen Eger, Gözde Gül Şahin, Andreas Rücklé, JiUng Lee, Claudia Schulz, Mohsen Mesgar, $\mathrm{Kr}$ ishnkant Swarnkar, Edwin Simpson, and Iryna Gurevych. 2019. Text processing like humans do: Visually attacking and shielding NLP systems. In Proceedings of NAACL-HLT.

Ji Gao, Jack Lanchantin, Mary Lou Soffa, and Yanjun Qi. 2018. Black-box generation of adversarial text sequences to evade deep learning classifiers. In Proceedings of IEEE Security and Privacy Workshops.

Siddhant Garg and Goutham Ramakrishnan. 2020. Bae: Bert-based adversarial examples for text classification. In Proceedings of EMNLP.

Ian J Goodfellow, Jonathon Shlens, and Christian Szegedy. 2015. Explaining and harnessing adversarial examples. In Proceedings of ICLR.

Dou Goodman, Hao Xin, Wang Yang, Wu Yuesheng, Xiong Junfeng, and Zhang Huan. 2020. Advbox: a toolbox to generate adversarial examples that fool neural networks. arXiv preprint arXiv:2001.05574.

Sepp Hochreiter and Jürgen Schmidhuber. 1997. Long short-term memory. Neural Computation, 9(8):1735-1780.

Mohit Iyyer, John Wieting, Kevin Gimpel, and Luke Zettlemoyer. 2018. Adversarial example generation with syntactically controlled paraphrase networks. In Proceedings of the NAACL-HLT.

Paul Jaccard. 1912. The distribution of the flora in the alpine zone.1. New Phytologist, 11(2):37-50. 
Robin Jia and Percy Liang. 2017. Adversarial examples for evaluating reading comprehension systems. In Proceedings of EMNLP.

Di Jin, Zhijing Jin, Joey Tianyi Zhou, and Peter Szolovits. 2020. Is BERT really robust? Natural language attack on text classification and entailment. In Proceedings of AAAI.

Rafal Jozefowicz, Oriol Vinyals, Mike Schuster, Noam Shazeer, and Yonghui Wu. 2016. Exploring the limits of language modeling. arXiv preprint arXiv:1602.02410.

Vladimir I Levenshtein. 1966. Binary codes capable of correcting deletions, insertions, and reversals. In Soviet physics doklady, volume 10, pages 707-710.

Jinfeng Li, Shouling Ji, Tianyu Du, Bo Li, and Ting Wang. 2019. Textbugger: Generating adversarial text against real-world applications. In Proceedings of Network and Distributed Systems Security Symposium.

Linyang Li, Ruotian Ma, Qipeng Guo, Xiangyang Xue, and Xipeng Qiu. 2020. Bert-attack: Adversarial attack against bert using bert. In Proceedings of EMNLP.

Paul Michel, Xian Li, Graham Neubig, and Juan Pino 2019. On evaluation of adversarial perturbations for sequence-to-sequence models. In Proceedings NAACL-HLT.

John Morris, Eli Lifland, Jin Yong Yoo, Jake Grigsby, Di Jin, and Yanjun Qi. 2020. Textattack: A framework for adversarial attacks, data augmentation, and adversarial training in nlp. In Proceedings of EMNLP: System Demonstrations.

Maria-Irina Nicolae, Mathieu Sinn, Minh Ngoc Tran, Beat Buesser, Ambrish Rawat, Martin Wistuba, Valentina Zantedeschi, Nathalie Baracaldo, Bryant Chen, Heiko Ludwig, et al. 2018. Adversarial robustness toolbox v1. 0.0. arXiv preprint arXiv:1807.01069.

Nicolas Papernot, Fartash Faghri, Nicholas Carlini, Ian Goodfellow, Reuben Feinman, Alexey Kurakin, Cihang Xie, Yash Sharma, Tom Brown, Aurko Roy, Alexander Matyasko, Vahid Behzadan, Karen Hambardzumyan, Zhishuai Zhang, Yi-Lin Juang, Zhi Li, Ryan Sheatsley, Abhibhav Garg, Jonathan Uesato, Willi Gierke, Yinpeng Dong, David Berthelot, Paul Hendricks, Jonas Rauber, and Rujun Long. 2018. Technical report on the cleverhans v2.1.0 adversarial examples library. arXiv preprint arXiv:1610.00768.

Nicolas Papernot, Patrick McDaniel, Somesh Jha, Matt Fredrikson, Z Berkay Celik, and Ananthram Swami. 2016a. The limitations of deep learning in adversarial settings. In 2016 IEEE European symposium on security and privacy (EuroS\&P). IEEE.
Nicolas Papernot, Patrick McDaniel, Ananthram Swami, and Richard Harang. 2016b. Crafting adversarial input sequences for recurrent neural networks. In Proceedings of MILCOM.

Kishore Papineni, Salim Roukos, Todd Ward, and WeiJing Zhu. 2002. Bleu: a method for automatic evaluation of machine translation. In Proceedings of $A C L$

Alec Radford, Jeffrey Wu, Rewon Child, David Luan, Dario Amodei, and Ilya Sutskever. 2019. Language models are unsupervised multitask learners. OpenAI Blog, 1(8).

Jonas Rauber, Wieland Brendel, and Matthias Bethge. 2017. Foolbox: A python toolbox to benchmark the robustness of machine learning models. In ICML Reliable Machine Learning in the Wild Workshop.

Nils Reimers and Iryna Gurevych. 2019. Sentencebert: Sentence embeddings using siamese bertnetworks. In Proceedings of EMNLP.

Shuhuai Ren, Yihe Deng, Kun He, and Wanxiang Che. 2019. Generating natural language adversarial examples through probability weighted word saliency. In Proceedings of ACL.

Marco Tulio Ribeiro, Sameer Singh, and Carlos Guestrin. 2018. Semantically equivalent adversarial rules for debugging NLP models. In Proceedings of $A C L$

Richard Socher, Alex Perelygin, Jean Wu, Jason Chuang, Christopher D Manning, Andrew Ng, and Christopher Potts. 2013. Recursive deep models for semantic compositionality over a sentiment treebank. In Proceedings of EMNLP.

Christian Szegedy, Wojciech Zaremba, Ilya Sutskever, Joan Bruna, Dumitru Erhan, Ian Goodfellow, and Rob Fergus. 2014. Intriguing properties of neural networks. In Proceedings of ICLR.

Eric Wallace, Shi Feng, Nikhil Kandpal, Matt Gardner, and Sameer Singh. 2019a. Universal adversarial triggers for attacking and analyzing NLP. In Proceedings of EMNLP-IJCNLP.

Eric Wallace, Jens Tuyls, Junlin Wang, Sanjay Subramanian, Matt Gardner, and Sameer Singh. 2019b. Allennlp interpret: A framework for explaining predictions of nlp models. In Proceedings of EMNLPIJCNLP.

Yuan Zang, Fanchao Qi, Chenghao Yang, Zhiyuan Liu, Meng Zhang, Qun Liu, and Maosong Sun. 2020. Word-level textual adversarial attacking as combinatorial optimization. In Proceedings of ACL.

Huangzhao Zhang, Hao Zhou, Ning Miao, and Lei Li. 2019. Generating fluent adversarial examples for natural languages. In Proceedings of ACL. 
Wei Emma Zhang, Quan Z Sheng, Ahoud Alhazmi, and Chenliang Li. 2020. Adversarial attacks on deep-learning models in natural language processing: A survey. ACM Transactions on Intelligent Systems and Technology (TIST), 11(3):1-41.

Zhengli Zhao, Dheeru Dua, and Sameer Singh. 2018. Generating natural adversarial examples. In Proceedings of ICLR. 